
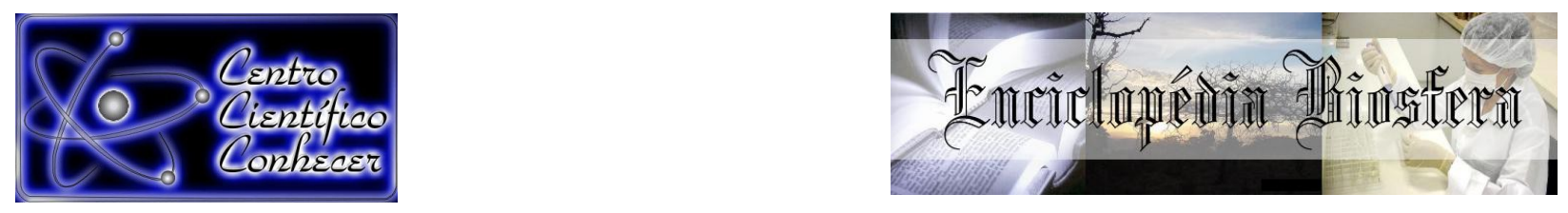

\title{
AVALIAÇÃO DA MANUTENÇÃO DA QUALIDADE DE UVAS PASSA BRS VITÓRIA APÓS APLICAÇÃO DE PRÉ-TRATAMENTO QUÍMICO
}

\author{
Vanessa Ferreira Caldeira', Sabrina Moura Guimarães ${ }^{1}$, Sérgio Tonetto de Freitas ${ }^{2}$, \\ Rita de Cássia Mirela Resende Nassur ${ }^{3}$ \\ 1 Discentes - Graduandas em Engenharia de Bioprocessos e Biotecnologia \\ Universidade do Estado da Bahia - Uneb - Departamento de Tecnologia e Ciências \\ Sociais - Campus III. \\ 2. Pesquisador, PhD Plant Biology. Embrapa Semiárido \\ 3- Docente, Dra Ciência dos Alimentos. Universidade do Estado da Bahia - Uneb - \\ Departamento de Tecnologia e Ciências, Sociais - Campus III. E-mail: \\ rnassur@uneb.br \\ Recebido em: 06/04/2018 - Aprovado em: 10/06/2018 - Publicado em: 20/06/2018 \\ DOI: 10.18677/EnciBio_2018A13
}

\begin{abstract}
RESUMO
A uva é considerada uma matéria prima de grande relevância, pois, a partir do seu cultivo é possível à obtenção de diferentes produtos alimentícios processados. Porém, etapas utilizadas no processamento de frutas podem alterar a taxa de degradação de compostos nutricionais de interesse. O objetivo dessa pesquisa foi avaliar a manutenção de parâmetros de qualidade da uva passa 'BRS Vitória' submetida à pré-tratamento com hidróxido de sódio $(\mathrm{NaOH})$, durante o armazenamento por até 120 dias. O pré-tratamento com $\mathrm{NaOH}$ é utilizado para induzir a rachadura da casca visando acelerar o processo de desidratação das bagas durante a produção de uva passa. Três repetições de uvas 'BRS Vitória' foram imersas em solução de $\mathrm{NaOH} 1 \%$ em fervura por 30 segundos. Outra parcela das bagas foi inserida diretamente na estufa após sua sanitização e, após a secagem, foram transferidas porções de $30 \mathrm{~g}$ de uvas para cada embalagem de polestireno com capacidade para $100 \mathrm{~g}$. As amostras de uva passa foram avaliadas aos $0,30,60,90$ e 120 dias de armazenamento a $25^{\circ} \mathrm{C}$ para as variáveis: $\mathrm{pH}$, acidez titulável, cor $\left(L, a^{*}, b^{*}\right)$, sólidos solúveis, atividade antioxidante, fenólicos totais e antocianinas. No geral, a aplicação de pré-tratamento químico não resultou em mudanças significativas na maioria dos parâmetros de qualidade avaliados em uvas passa 'BRS Vitória'. Substâncias antioxidantes obtiveram maior manutenção de teores em uvas passa pré-tratadas durante a maior parte do experimento. De acordo com a análise multivariada, o tempo de armazenamento das uvas passa foi 0 principal fator influenciador das variáveis estudadas.
\end{abstract}

PALAVRAS-CHAVE: Antioxidantes, Armazenamento, Pré-tratamento com $\mathrm{NaOH}$. 


\title{
EVALUATION OF THE QUALITY MAINTENANCE OF 'BRS VITÓRIA' RAISINS AFTER CHEMICAL PRE-TREATMENT
}

\begin{abstract}
The grape is considered a fruit of great relevance due to the fact that it is possible to obtain different processed food products. However, the processing and storage of the fruit can affect the contents of nutritional and quality compounds. The objective of this study was to evaluate the quality maintenance of 'BRS Vitória' raisins submitted to pre-treatment with sodium hydroxide $(\mathrm{NaOH})$ during storage for a period of 120 days. Pretreatment with $\mathrm{NaOH}$ is used to induce peeling crack, which accelerates the berry dehydration during raisin production. Three repetitions of 1200 grams of 'BRS Vitória' grapes were immersed in a boiling $\mathrm{NaOH}$ solution for 30 seconds. Other three repetitions of 650 grams were placed directly into the oven at $60^{\circ} \mathrm{C} \pm 5^{\circ} \mathrm{C}$ after its sanitization and, after drying, portions of $30 \mathrm{~g}$ were transferred to polystyrene packages (100 g storage capacity). Raisins samples were evaluated at 0, 30, 60, 90 and 120 days storage at $25^{\circ} \mathrm{C}$ for $\mathrm{pH}$, titratable acidity, color $\left(\mathrm{L}^{*}, \mathrm{a}^{*}\right.$ and $\left.\mathrm{b}^{*}\right)$, soluble solids, antioxidant activity, total phenolics and anthocyanins. The application of chemical pretreatment did not resulted in $\mathrm{pH}$, titratable acidity, color $\left(L, a^{*}, b^{*}\right)$, soluble solids, antioxidant activity, total phenolics and anthocyanins significant changes in most of the evaluations of 'BRS Vitoria' raisins. Antioxidant substances were observed in higher amounts in treated fruits during the experiment evaluations. According to the multivariate analysis, storage time was the main influencing factor of the studied variables in raisins produced at the São Francisco River Valley, Brazil.
\end{abstract}

KEYWORDS: Antioxidants, Pretreatment with $\mathrm{NaOH}$, Storage.

\section{INTRODUÇÃO}

O Brasil é destaque na produção de frutas, tornando-se um dos maiores produtores mundiais (MACHADO et al., 2015; AMARAL et al., 2016). Uma das frutas que fazem parte da cadeia produtiva nacional é a uva (DEBASTIANI et al., 2015). No Nordeste, na região do Vale do Submédio do São Francisco, fatores característicos como condições de clima, solo e baixa umidade, além da utilização de sistema irrigado, beneficiam a produção de uva, evidenciando o potencial para o desenvolvimento de frutos de ótima qualidade durante todo o ano, pela possibilidade de mais de uma safra anual, tornando-se destaque nacional e internacional (SANTOS et al., 2011; SILVA et al., 2015).

A uva é considerada uma matéria prima de grande relevância, pois, a partir do cultivo é possível à obtenção de produtos alimentícios processados que apresentam, ainda, caráter funcional, em virtude, do seu teor de antioxidantes, que podem ser incorporados na dieta (LINS; SARTORI, 2014; VILAS BOAS et al., 2016). Nas últimas décadas diversas pesquisas ressaltam os efeitos positivos do consumo de 
alimentos ricos nesses componentes, que são considerados promotores da saúde humana (ACHKAR et al., 2013).

O processamento de frutos in natura com a finalidade de transformá-los em produtos diferenciados permite a obtenção de alimentos com alto valor agregado, como por exemplo, a obtenção de frutas secas, que são produtos com demanda cada vez maior, estando à uva passa inserida nesse cenário (SOUZA et al., 2015). O consumo de uvas passa no Brasil é alto devido as suas vantagens nutricionais, proporcionando saúde e bem estar e, além disso, podem ser incorporadas como ingrediente adicional em outros alimentos como, barras de cereais, bolos, sorvetes, iogurtes, entre outros (JESZKA-SKOWRON et al., 2017).

A produção nacional desse produto é muito baixa, isso ocorre, principalmente, devido à pequena aplicação de áreas para o cultivo de uvas sem sementes, além do que a maior parte das safras destina-se a elaboração de vinhos, sucos e uva de mesa (FREITAS et al., 2013; AMARAL et al., 2016). Grande parte da uva passa consumida no país é, portanto, importada de outros países.

Algumas frutas, como a uva, possuem um revestimento ceroso que limita a permeabilidade da casca, dificultando o deslocamento da água do interior do fruto para o meio externo (TARHAN, 2007). Assim, antes de serem expostas ao processo de secagem, as uvas podem ser submetidas a alguns pré-tratamentos químicos, de forma a promover o aumento da permeabilidade e otimizar a desidratação, preservando a qualidade do produto final (GABAS et al., 1998).

Diferentes tipos de pré-tratamento químico são utilizados para conferir a quebra da impermeabilidade da casca da uva, estas são comumente imersas em soluções que contêm hidróxido de sódio ( $\mathrm{NaOH}$ ) (CARRANZA-CONCHA et al., 2012), carbonato de potássio $\left(\mathrm{K}_{2} \mathrm{CO}_{3}\right)$ (BINGOL et al., 2012) e carbonato de cálcio $\left(\mathrm{CaCO}_{3}\right)$ (GABAS et al., 1999). Porém, além de notar-se o benefício da aplicação dessas substâncias com relação ao tempo de secagem é preciso levar em consideração que a utilização de diferentes soluções com conteúdo químico pode prejudicar aspectos nutricionais dos produtos diminuindo teores de alguns parâmetros desejáveis, como o conteúdo de antioxidantes (CARRANZA-CONCHA et al., 2012).

Diante dos potenciais benefícios que esse tipo de alimento pode proporcionar é de suma importância à viabilização da manutenção de compostos de interesse nutricional durante o processamento e armazenamento da uva passa. Assim, é necessário levar em consideração que a utilização de pré-tratamentos químicos pode influenciar a concentração de compostos de interesse. Além disso, tempo de armazenamento e o material de embalagens também são fatores que devem ser levados em conta ao avaliar o potencial nutricional e a qualidade de produtos processados (JAVED et al., 2018).

A cultivar de uva BRS Vitória é uma variedade que faz parte do programa de melhoramento genético da Empresa Brasileira de Pesquisa Agropecuária (Embrapa). Esta cultivar apresenta bagas de coloração negra e alta fertilidade de gemas, cachos levemente compactos, bagas esféricas e pequenas e película grossa. Devido às características de qualidade, estudos preliminares têm identificado a cultivar BRS Vitória como um genótipo com alto potencial para a produção de uvas passa no Vale do São Francisco (FREITAS et al., 2013). 
Diante disso, esse trabalho teve como objetivo avaliar a manutenção de parâmetros de qualidade da uva passa 'BRS Vitória' submetida à pré-tratamento com hidróxido de sódio ( $\mathrm{NaOH}$ ) e fazer uma comparação com uvas não expostas a esse tipo de tratamento, durante seu armazenamento por um período de 120 dias.

\section{MATERIAL E MÉTODOS}

Uvas sem semente 'BRS Vitória' foram colhidas no estádio de maturação utilizado para o consumo in natura e foram desengaçadas e lavadas em água corrente. Seiscentos e cinquenta gramas das bagas foram inseridas diretamente na estufa após essa etapa. O restante dos frutos $(1200 \mathrm{~g})$ foram imersos em uma solução de hidróxido de sódio ( $\mathrm{NaOH} 1 \%$ ), em fervura, durante 30 segundos, de modo a facilitar a quebra da impermeabilidade da casca, em seguida as bagas foram lavadas em água corrente para retirar o excesso da solução. Os frutos foram pesados e colocados em bandejas para secagem a $60^{\circ} \mathrm{C} \pm 5^{\circ} \mathrm{C}$, em estufa modelo TE-394/2 (Tecnal, Piracicaba, SP, Brasil), com circulação forçada de ar. Durante esse período (cerca de 72 horas), os frutos foram revolvidos nas bandejas, a cada 12 horas para a homogeneização da secagem.

As uvas passa foram secas até que a atividade de água dos frutos atingisse o valor de 0,4. Após a secagem, foram preparadas porções de $30 \mathrm{~g}$ de uvas em embalagens de polestireno com tampa (capacidade para $100 \mathrm{~g}$ ). Cada tratamento e tempos de armazenamento foram representados por três repetições. As amostras de uva passa foram avaliadas aos $0,30,60,90$ e 120 dias de armazenamento a $25^{\circ} \mathrm{C}$.

As uvas foram avaliadas no seu estado in natura, antes do processo de secagem quanto a sua firmeza, sólidos solúveis, acidez total, $\mathrm{pH}$ e coloração. As avaliações durante a vida útil foram: $\mathrm{pH}$, realizado por potenciometria de acordo com AOAC (2012) em titulador com potenciômetro modelo Titrino plus 848 (Metrohm, São Paulo, SP, Brasil); acidez titulável, determinada por titulometria de acordo com a metodologia sugerida pela AOAC (2012) com resultados expressos em \% de ácido tartárico; cor, avaliada com o auxílio de um colorímetro modelo CR-400 (Minolta, Piracicaba, SP, Brasil), sendo os resultados expressos nas escalas $L$, $a^{*}$ e $b^{*}$ no sistema CIELab; sólidos solúveis (SS) foram obtidos por refratometria em refratômetro modelo PAL-1 (ATAGO, Ribeirão Preto, SP, Brasil); atividade antioxidante foi determinada a partir do método do 2,2-difenil-1-picril-hidrazil (DPPH) de acordo com Rufino et al. (2007); fenólicos totais foram determinados por meio de espectroscopia na região do visível utilizando o método de Folin-Ciocalteu com modificações (SINGLETON et al., 1999); antocianinas foram quantificadas de acordo com a metodologia de Giusti e WrolsItad (2001).

Os resultados obtidos foram submetidos à análise de variância (ANOVA). O fator quantitativo tempo de armazenamento foi submetido à análise de regressão e o fator qualitativo pré-tratamento (com e sem $\mathrm{NaOH}$ ) foi submetido à análise pelo teste de Tukey $(5 \%)$ com auxílio do software SISVAR ${ }^{\circledR}$. Além disso, para otimizar a visualização dos resultados, foi realizada uma análise estatística multivariada (análise de componentes principais - ACP) através do software $\mathrm{XLStat}^{\circledR}$. 


\section{RESULTADOS E DISCUSSÃO}

As uvas avaliadas no seu estado in natura, antes do processo de secagem e do pré-tratamento com $\mathrm{NaOH}$ apresentaram, em média, $26,3 \%$ de sólidos solúveis, $0,52 \%$ de acidez total, e pH de 3,77 . Quanto ao parâmetro cor, a luminosidade $\left(L^{*}\right)$ observada foi 22,48, a coordenada $a^{*}-1,28$ e $b^{*} 0,99$. Para firmeza obteve-se 385,22 Kgf. Os valores observados no presente trabalho podem ser considerados ideais quando comparados com os determinados pela legislação (BRASIL, 2000). Machado et al. (2015), em estudo com a variedade de uva Isabel para obtenção de uva passa, ao caracterizarem o fruto in natura obtiveram valores de $12,09 \%$ para sólidos solúveis, $0,76 \%$ para acidez total e 3,51 para $\mathrm{pH}$. As uvas passa obtidas, sem e com o pré-tratamento com $\mathrm{NaOH}$, apresentaram, respectivamente, umidade média de $77,9 \%$ e $73,6 \%$, e atividade de água de 0,4 . O pré-tratamento com $\mathrm{NaOH}$ resultou em rompimento das cascas.

A coloração é um atributo de qualidade importante, tanto para frutos in natura quanto para processados, pois é bastante relevante para a aceitação dos alimentos pelos consumidores, sendo o aspecto visual o primeiro fator decisivo durante a escolha do produto (RIBEIRO et al., 2012). O parâmetro de cor $L^{*}$ representa a luminosidade do produto e pode variar do preto (0) ao branco (100). De acordo com os resultados obtidos, o aumento do tempo de armazenamento intensificou o escurecimento das uvas passa, com variações durante o período experimental de 18,4 a 22,00 . Porém, não foi observado efeito significativo na luminosidade de escurecimento das uvas passas quanto ao pré-tratamento empregado (Figura 1).

Ao avaliar perfis de coloração em frutos, outro parâmetro a ser considerado é a coordenada $a^{*}$, que indica a cor para tons de vermelho e verde, esta foi influenciada apenas pelo tempo de armazenamento, independentemente do tipo de tratamento utilizado, apresentando um valor médio de 2,24, obtendo-se valores maiores no início do experimento $(3,01)$ e menores no tempo $30(1,38)$ (Figura 1). Os resultados positivos para $a^{*}$ são um indicativo da predominância da cor vermelha sobre a verde, indicando que esse tom estava mais presente nas amostras de uva passa e variou de acordo com o tempo. Silva et al. (2015), em experimento com uva passa da variedade Crimson, atingiram, para a coordenada $a^{*}$ valor de, aproximadamente, 3,8 após secagem a $70^{\circ} \mathrm{C}$, apontando, também, a predominância da cor vermelha.

A cor dos alimentos está associada, principalmente, à presença de pigmentos naturais. Portanto, essa variável pode estar relacionada com a existência de antocianinas, que são pigmentos responsáveis por cores como, roxo e diferentes tons de vermelho em frutos, caules e folhas (BUCKOW et al., 2010). Esses elementos são instáveis, assim, fatores como processamento do fruto, condições e tempo de armazenamento podem ocasionar a sua degradação; a temperatura, a luz, e a presença de oxigênio são variáveis que influenciam no processo de deterioração das antocianinas, provocando, assim, mudançanças de cor (SCHIOZER; BARATA, 2007).

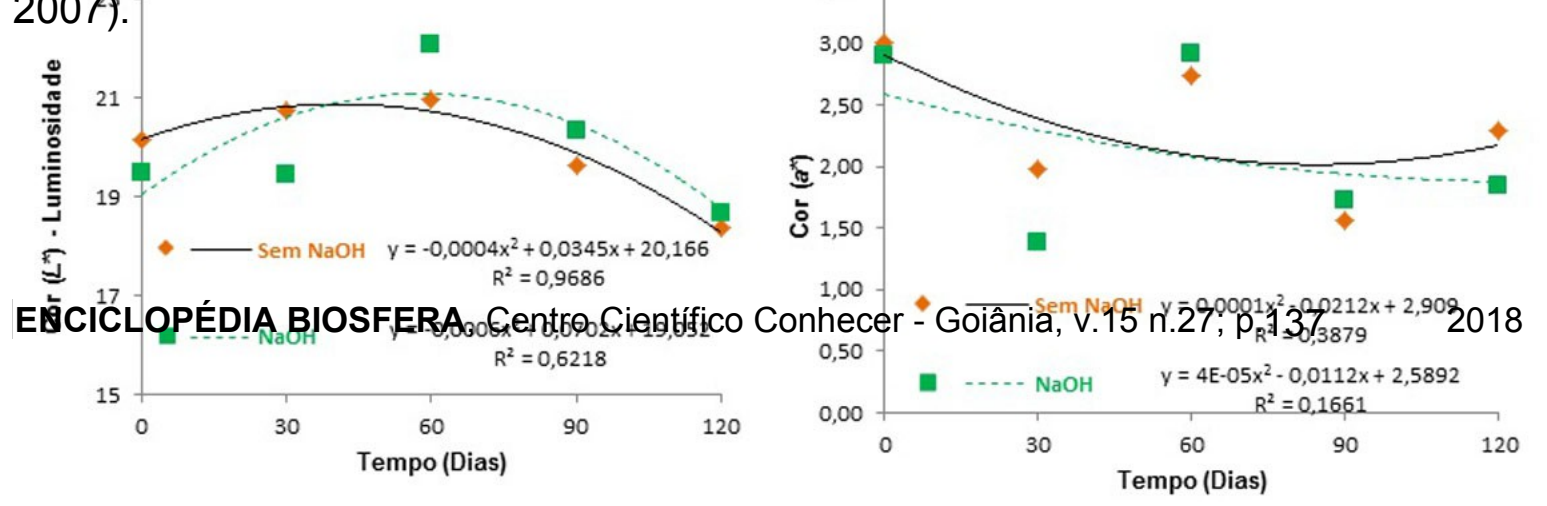


FIGURA 1. Luminosidade $\left(L^{*}\right)$ e coordenada $a^{*}$ (vermelho/verde) de uvas passa sem e com pré-tratamento com $\mathrm{NaOH}$ armazenadas por até 120 dias.

A variável $b^{*}$ reflete a mudança de tons entre o amarelo e azul, em que menores valores simbolizam uma coloração mais azulada dos frutos. Não foi observado efeito significativo quanto aos tratamentos aplicados durante os 120 dias. Os valores médios de $b^{*}$ verificados foram de 1,5 durante 0 tempo de armazenamento.

A coloração é um dos fatores que determinam a presença de um alimento no mercado (SANTOS et al., 2011). Segundo Rocha e Reed (2014), a cor é determinada pela presença de pigmentos, e, alguns destes podem ter papel significativo para saúde. Os flavonoides, por exemplo, fazem parte de um grupo de pigmentos, e são subdivididos em fenólicos, antocianinas e outros, estes, são pigmentos bastante presentes nas uvas tintas, responsáveis pela cor de suas cascas. Além do fator nutricional, esses tipos de substâncias estão intensamente relacionados com uma melhor percepção visual dos produtos comercializados.

Quanto à firmeza, observou-se que ocorreram diferenças significativas para tempo de armazenamento e entre os dois tipos de tratamento pelos quais as passas foram submetidas. Esse parâmetro variou entre valores médios de 3,87 $\mathrm{N}$ (tempo 0) e 1,37 N (tempo 60). Quando avaliado quanto ao tipo de tratamento às uvas apresentaram, em média, 1,82 N (sem NaOH) e 2,82 N (com NaOH) (Figura 2). A quebra da impermeabilidade da casca da uva (com $\mathrm{NaOH}$ ) deixa-as mais expostas a temperatura por tempo prolongado e com isso pode ter ocorrido efeitos sobre a firmeza das passas.

Relaciona-se a firmeza à força necessária para promover uma deformidade, através da compressão manual ou bucal (MASCARENHAS et al., 2010). A mastigabilidade e a firmeza de frutos é um parâmetro de qualidade de alimentos que o consumidor julga na hora da compra e deve ser considerado, em testes de qualidade de produtos processados. De acordo com a análise sensorial de uvas passa de diferentes variedades, feita e apresentada no trabalho de Freitas et al. (2013), para a maioria dos avaliadores a preferência pelo consumo de uvas passa foi determinada entre aquelas que possuem doçura, suculência e maciez aquelas que se apresentaram com características de dureza e ressecamento foram rejeitadas. Rybka et al. (2015), em um experimento envolvendo diferentes condições 
de secagem para uvas da variedade 'Itália', observaram valores de 4,2 $\mathrm{N}$ para firmeza de uvas passa após 48 horas, a $56^{\circ} \mathrm{C}$.
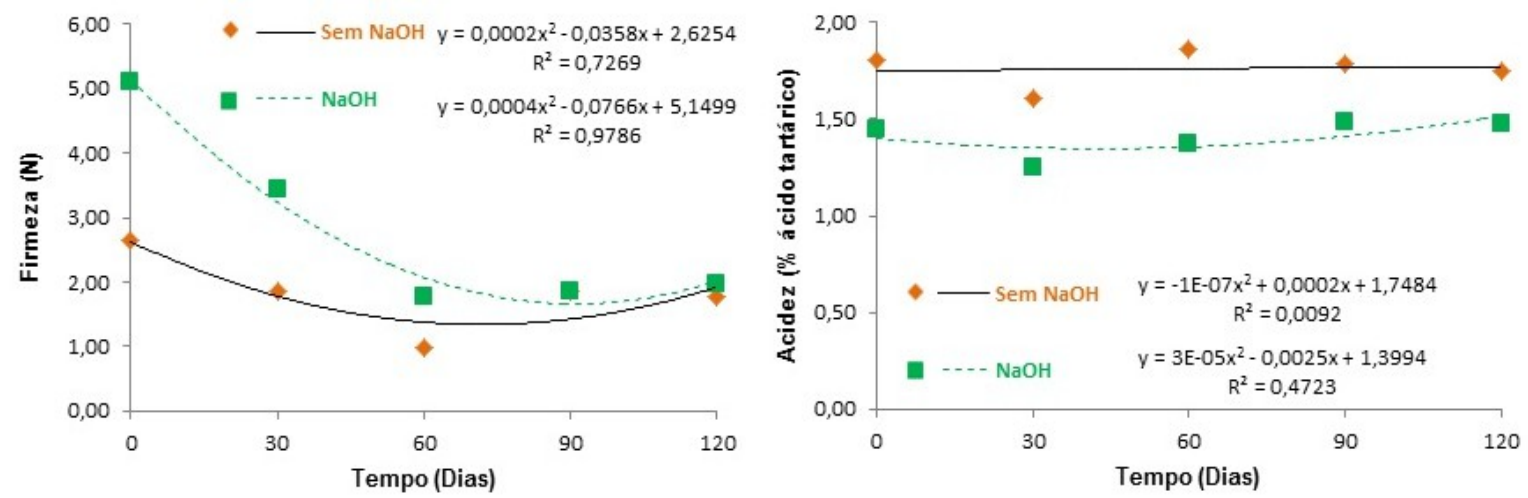

FIGURA 2. Firmeza e acidez (em \% de ácido tartárico) de uvas passa sem e com pré-tratamento com $\mathrm{NaOH}$ armazenadas por até 120 dias.

No geral, não foram observados efeitos significativos com relação ao tempo de armazenamento no teor de acidez das uvas passa BRS Vitória, que foram influenciadas somente pelo tipo de tratamento utilizado. Observou-se valores médios de $1,75 \%$ para uvas não tratadas com $\mathrm{NaOH}$ e de $1,41 \%$ de ácido tartárico para bagas submetidas ao pré-tratamento químico (Figura 2). Os constituintes predominantes dos sólidos solúveis da polpa da uva são os açúcares solúveis, no entanto, os ácidos orgânicos também são responsáveis para constituição do sabor desse fruto. Além disso, o teor de acidez é um parâmetro que pode indicar o estado de conservação de um produto alimentício, essa variável está relacionada à presença de ácidos encontrados naturalmente em alguns vegetais, como os ácidos málico, cítrico e tartárico, sendo esse último predominante na uva (RIBEIRO et al., 2016). Pesquisa feita por Carranza-Concha et al. (2012), revelou que, no geral, a acidez de uvas que foram submetidas a secagem após pré-tratamento com $\mathrm{NaOH}$ apresentaram perdas significativas, o que foi explicado pela diminuição de ácidos, como o ácido ascórbico, que, de acordo com os autores, podem ter sido causadas pela lixiviação durante o tempo de imersão das bagas e degradação do ácido em meio alcalino.

Costa et al. (2015), em pesquisa envolvendo a caracterização físico-química de uva 'Itália', produzidas no Vale do São Francisco, obtiveram, após desidratação a diferentes temperaturas, teor médio de acidez entre as faixas de 1,5 a 4,93\%. Em alimentos, essa variável está associada ao flavor, a deterioração pelo crescimento de micro-organismos, diminuição do escurecimento enzimático, redução da ação de substâncias redutoras.

O sabor e a qualidade sensorial de frutos são determinados pela soma de fatores como, doçura, adstringência, amargura, salinidade, compostos voláteis e acidez (GUINÉ et al., 2010). Esses atributos foram avaliados em trabalho feito por Mascarenhas et al. (2010), que avaliaram a aceitabilidade de consumidores por uvas de diferentes variedades e verificaram que, após as análises dos consumidores, foi possível concluir que uvas com maiores teores de sólidos solúveis e que possuam baixa acidez têm maior aceitação pelos usuários, destacando assim a importância desse parâmetro na comercialização desses frutos. 
Os valores de $\mathrm{pH}$ não foram influenciados pelo tratamento utilizado, apenas pelo tempo de armazenamento. Apesar de, no geral, ter ocorrido pouca variação, entre os tempos 30 e 60, principalmente, houve interações significativas para esse parâmetro (Tabela 1). Foi observado que o pico dos valores de $\mathrm{pH}$ aconteceu após os 60 dias de armazenamento, em média, 4,17, para ambos tratamentos. Valores próximos foram encontrados após a desidratação de uvas 'Itália' em estudo feito por Costa et al. (2015), resultando em passas com valores de pH entre 3,5 e 4,5.

TABELA 1. pH de uvas passa sem e com pré-tratamento com $\mathrm{NaOH}$ armazenadas por até 120 dias.

\begin{tabular}{lccccc}
\hline & 0 & 30 & 60 & 90 & 120 \\
\cline { 2 - 6 } Sem NaOH & $3,90^{a}$ & $2,97^{b}$ & $4,16^{a}$ & $3,89^{a}$ & $3,92^{a}$ \\
$\mathrm{NaOH}$ & $3,98^{\mathrm{a}}$ & $3,15^{\mathrm{b}}$ & $4,17^{\mathrm{a}}$ & $3,86^{\mathrm{a}}$ & $3,96^{\mathrm{a}}$ \\
*Letras iguais, na mesma linha, médias não diferem entre si pelo teste \\
de Skott Knott a 5\%.
\end{tabular}

Micro-organismos como, leveduras, bolores e bactérias, são sensíveis ao $\mathrm{pH}$ de um alimento. O potencial hidrogeniônico interfere consideravelmente no crescimento desses organismos, valores de $\mathrm{pH} \leq 4,5$, em média, evitam sua multiplicação, resultando em produtos mais seguros (SANTOS et al., 2011). Além disso, o $\mathrm{pH}$ pode estar associado a atividade de enzimas, sabor e estabilidade.

Dentre a variedade de componentes existentes na fruta, os sólidos solúveis também desempenham um papel importante para a sua qualidade, eles compreendem compostos solúveis na célula dos alimentos, que são responsáveis pelo sabor, como carboidratos e ácidos orgânicos. Essa variável apresenta a quantidade de açúcares e outros sólidos solúveis presentes na amostra (GOUVEIA et al., 2014). Como esperado para o presente estudo, a desidratação das uvas elevou a concentração de sólidos solúveis. O teor de sólidos solúveis não foi significativamente alterado pelo tempo de armazenamento e nem quando houve ou não pré-tratamento com $\mathrm{NaOH}$, observando-se valores médios iguais a $76,1 \%$, para uvas não pré-tratadas e 75,6 \% para uvas que passaram por pré-tratamento.

Costa et al. (2015), observaram teores equivalentes para essa variável de, aproximadamente, $75 \%$ em uvas passa da variedade Itália, produzidas na região do Vale do Submédio do São Francisco, após frutos serem expostos a secagem a $70^{\circ}$ $\mathrm{C}$, durante 54 horas.

$\mathrm{Na}$ figura 3 é possível observar os valores médios para a atividade antioxidante de uvas submetidas à pré-tratamento, armazenadas durante um período de até 120 dias. Durante o período experimental essa variável foi influenciada pelo tempo de armazenamento. Uvas que foram submetidas ao prétratamento químico conseguiram uma melhor manutenção da atividade antioxidante, para esse parâmetro, porém, observou-se que houve uma tendência a diminuição dos teores, principalmente ao final dos 120 dias $(94,63 \%$ a $80,27 \%$ de sequestro de radicais livres).

Os valores observados para essa variável indicam que mesmo após o decréscimo destes eles permaneceram em níveis consideráveis de conteúdo ENCICLOPÉDIA BIOSFERA, Centro Científico Conhecer - Goiânia, v.15 n.27; p.140 2018 
antioxidante, e nesse sentido são equivalentes a outros produtos que também apresentam compostos que possuem essa característica e podem apresentar potencial para proporcionar benefícios quando consumidos. Nicolosi et al. (2018), ao avaliarem a vida útil de uvas de mesa observaram que, durante o armazenamento, ocorreu uma diminuição significativa da atividade antioxidante, porém, ao final desse período, os níveis de atividade antioxidante das uvas permaneceram eficientes. Vilas Boas et al. (2016), após verificarem o sequestro de radicais livres em blends de sucos de uva observaram valores médios de $32,4 \%$ durante 120 dias de armazenamento. Esses trabalhos evidenciam que esse tipo de composto bioativo tem uma tendência ao declínio durante sua vida útil.

Além do pré-tratamento e tempo de armazenamento, outros fatores podem interferir para decréscimos de substâncias antioxidantes, como por exemplo, a temperatura e o material de embalagem aos quais os frutos foram acondicionados (JAVED et al., 2018). Carneiro et al. (2016), após avaliarem a estabilidade de geleias de amora-preta acondicionadas em diferentes embalagens, observaram que a atividade antioxidante foi influenciada pelo tipo de embalagem e tempo de armazenamento, os resultados apontaram que geleias mantidas em embalagens de polipropileno demonstraram teores inferiores $(62,32$ \% SRL) para esse parâmetro.

A atividade antioxidante em vegetais está associada à diversidade de compostos antioxidantes que são degradados ou mantidos conforme o estado fisiológico e fatores abióticos e bióticos durante o armazenamento (ROTILI et al., 2013). De acordo com Zhang e Tsao (2016), essas substâncias estão presentes em alguns tipos de alimentos e são importantes para prevenção de doenças crônicas e contribuem para minimizar o estresse oxidativo, que ocorre nas reações metabólicas, por exemplo. Assim, o consumo regular de quantidade adequada de frutos secos pode reduzir a glicemia e fatores de risco cardiovasculares. As passas ajudam a manter a boa saúde e desempenham um papel importante na imunidade contra diferentes doenças (JAVED et al., 2018).
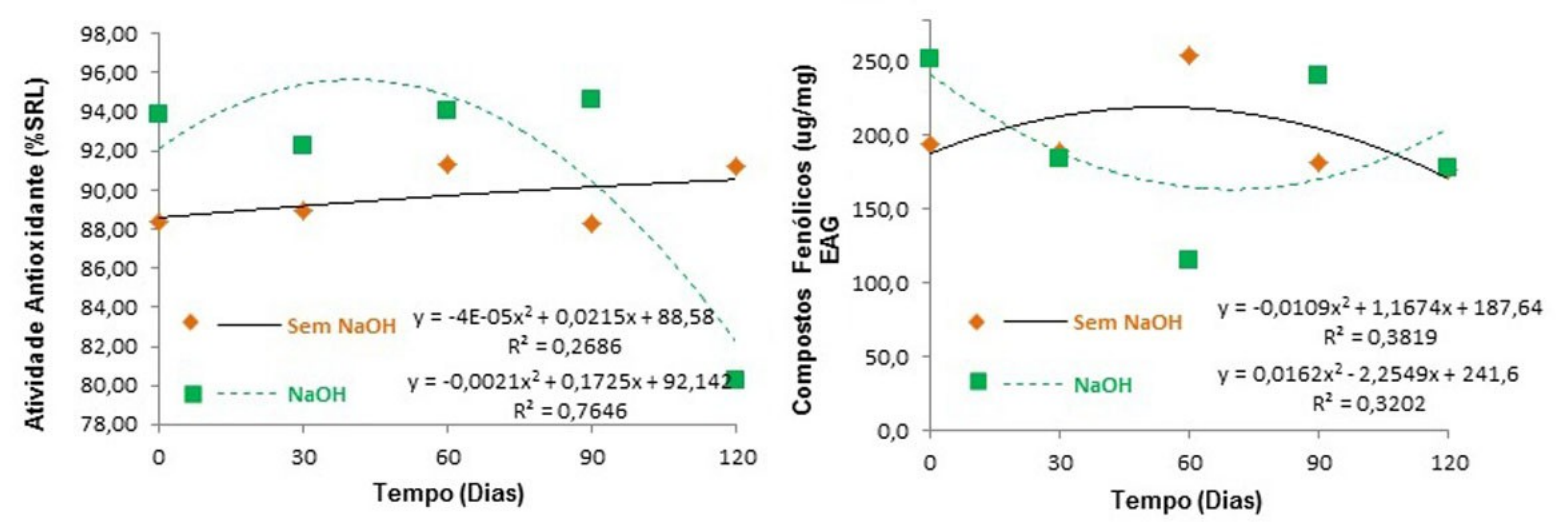
FIGURA 3. Atividade antioxidante (\% sequestro de radicais livres) e compostos Fenólicos $(\mu \mathrm{g} / \mathrm{mg})$ EAG de uvas passa sem e com pré-tratamento com $\mathrm{NaOH}$ armazenadas por até 120 dias.

Com relação ao teor de fenólicos em uvas passa da variedade BRS Vitória, foi observada interação significativa entre os fatores estudados (tempo de armazenamento e pré-tratamento). Observou-se maior manutenção de teores de compostos fenólicos em uvas passa não expostas ao pré-tratamento químico, onde resultaram, em média, 198,8 (ug/mg) EAG, destacando-se as passas após os 60 dias $(254,1 \mu \mathrm{m} / \mathrm{mg}$ EAG). Para bagas submetidas ao $\mathrm{NaOH}$ antes da secagem em estufa, o início do armazenamento foi o ponto mais expressivo, atingindo 252,1(ug/mg) EAG (Figura 3).

O processamento de uvas passa pode ocasionar a perda de frações fenólicas resultantes da oxidação enzimática e do ar que ocorre durante o processo (SÉRIO et al., 2014). A redução quantitativa desse tipo de substância é algo comum, também, em outros trabalhos que envolvem subprodutos da uva, uma vez que são compostos que possuem sensibilidade a diferentes fatores aos quais esses produtos são expostos durante o armazenamento como, luz, oxigênio e calor, por exemplo. Vilas Boas et al. (2016), ao avaliarem o teor de fenólicos em blends de sucos de uvas produzidas no sudoeste de Minas Gerais, obtiveram valores médios de 257,12 mg.100 ml-1 para um blend Niágara x BRS Cora x BRS Violeta durante 120 dias de armazenamento, observaram também que, durante esse tempo, houve redução desses bioativos.

A associação entre antioxidantes e fenólicos com a saúde torna a estabilidade desses compostos algo importante durante o processamento e armazenamento de alimentos que os contêm. Essas substâncias são significativas porque atuam na promoção da saúde, e o consumo regular de uvas e vinhos, ricos nesses compostos, é associado à baixa incidência de doenças cardiovasculares (LINS; SARTORI, 2014).

Uvas expostas a diferentes tipos de tratamento se diferenciaram significativamente quanto aos teores de antocianinas. Durante o início do experimento bagas que foram submetidas à solução com $\mathrm{NaOH}$, atingiram 48,99 $\mathrm{mg} / \mathrm{L}$ para antocianinas, enquanto para a parcela sem $\mathrm{NaOH}$ os teores foram equivalentes a $38,61 \mathrm{mg} / \mathrm{L}$ (Figura 4). Notou-se que o tempo de armazenamento também foi um fator influenciador no conteúdo dessas substâncias, resultando em diminuição durante esse período. As variações observadas durante o período experimental podem ter ocorrido devido à tendência dessas substâncias bioativas a sofrerem degradação como resposta a variações de temperatura e condições de armazenamento. Alguns estudos que envolvem produtos processados que possuem substâncias como essa relatam diferenças significativas entre teores iniciais e finais de antocianinas (JUNIOR et al., 2016). 


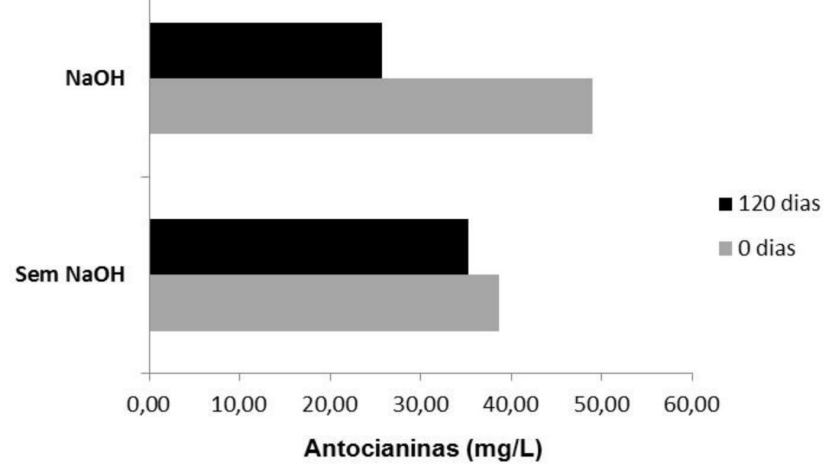

FIGURA 4. Antocianinas em uvas passa sem e com pré-tratamento com $\mathrm{NaOH}$ armazenadas por até 120 dias..

No decorrer do processamento e armazenamento, frutos secos podem ser submetidos a diversas condições, como alterações de temperatura, luminosidade, umidade, oxigênio, e a partir daí dar início a mudanças físico-químicas que podem ser indesejáveis (UDOMKUN et al., 2016). A alteração da coloração de um alimento, por exemplo, é resultado das alterações químicas e bioquímicas que podem ocorrer durante o processamento e estocagem. As antocianinas são pigmentos naturais presentes em frutos como a uva, eles são responsáveis pela caracterização da cor, a manutenção e a integridade dessas substâncias são influenciadas principalmente pela luz e temperatura (GUINÉ et al., 2010). A permeabilidade em embalagens também é uma condição que pode interferir no conteúdo de antocianinas, pois, a existência de oxigênio nesse meio é um dos fatores para degradação de antocianinas, podendo acontecer por meio da oxidação, independentemente da presença ou ausência de luz (CARNEIRO et al., 2016).

Quimicamente, essas substâncias são compostos fenólicos, da família dos flavonoides, suas propriedades funcionais contribuem para a agregação de valor ao produto final, pois são importantes na dieta humana auxiliando na prevenção de doenças cardiovasculares e do câncer devido a seu potencial de combate aos radicais livres, além de reduzir os níveis de colesterol e triglicerídeos (SANTOS et al., 2014). Esses bioativos podem ser inseridos na dieta a partir da ingestão de frutas como a uva, cereja, maçã, amora, entre outros ou a partir de alimentos enriquecidos nutricionalmente (SANTOS et al., 2014). Estudo feito por Bonfietti (2017), comprovou o incremento do valor nutricional através da adição desses pigmentos naturais, provenientes de açaí em pó, em cookies.

A partir da estatística multivariada - análise de componentes principais (ACP) -, que explicou $58,70 \%$ dos resultados, foi possível correlacionar as diferentes variáveis e tratamentos estudados. No gráfico (Figura 5), é possível observar que o tempo de armazenamento teve uma maior influência sobre os resultados em detrimento do pré-tratamento químico utilizado. Isso se deve ao fato de que há proximidade entre o mesmo tempo de armazenamento, independente do tratamento ao qual foi submetido. Os vetores que representam o resultado para luminosidade $e$ atividade antioxidante encontram-se próximos, indicando uma correlação positiva entre essas variáveis que podem estar relacionadas através das antocianinas, pigmentos responsáveis pela coloração dos frutos. O contrário pode ser observado 
para as $\mathrm{pH}$ e firmeza, a posição em que localizam-se os vetores para essas variáveis, de forma oposta, sugere uma correlação negativa entre ambas, apontando que não houve influência de uma sobre a outra. A relação direta entre $\mathrm{pH}$ e acidez é algo que também fica evidente no gráfico, através da posição dos vetores que representam essas variáveis.

Uvas passas armazenadas por 120 dias apresentaram-se positivamente correlacionadas com a acidez, um dos parâmetros responsáveis pela constituição do sabor em alimentos e seu estado de conservação. É possível concluir, ainda que frutos submetidos ao pré-tratamento com $\mathrm{NaOH}$ mostraram-se menos ácidos quando comparados aqueles que não passaram por esse tipo de processamento.

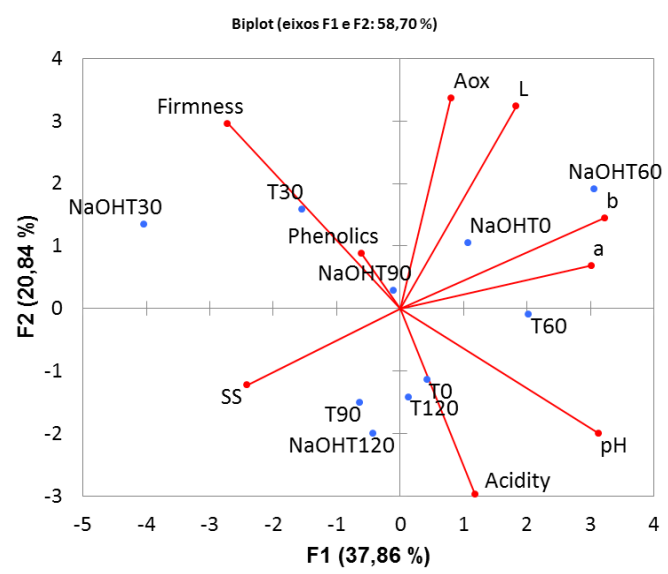

FIGURA 5. Análise dos componentes principais $(\mathrm{ACP})$ de uvas passa sem e com pré-tratamento com $\mathrm{NaOH}$ armazenadas por até 120 dias.

A hipótese testada considera que diferentes tipos de pré-tratamento influenciam na manutenção de compostos de interesse de uvas passa. Era esperado que uvas pré-tratadas com $\mathrm{NaOH}$ resultassem em menor tempo de secagem, devido a quebra da impermeabilidade da casca, o que ocasionaria a otimização da desidratação. Porém não foi observado efeito significativo nem para redução do tempo de secagem nem para maioria das variáveis estudadas.

\section{CONCLUSÕES}

No geral, a aplicação de pré-tratamento químico não provocou mudanças significativas na maioria dos parâmetros de qualidade avaliados em uvas passa BRS Vitória. Substâncias antioxidantes (compostos fenólicos e antocianinas) obtiveram maior manutenção de teores em uvas passa pré-tratadas durante a maior parte do experimento, porém ao final do armazenamento esses valores diminuem de forma significativa.

Além disso, o sabor das passas pode ser afetado conforme o declínio de parâmetros como a acidez, fato observado em bagas pré-tratadas com $\mathrm{NaOH}$, pois há relação com ácidos orgânicos, que são responsáveis pela constituição do sabor do fruto. 
Assim, fica evidente que para bagas que precisarão ser armazenadas em longo prazo esse tipo de tratamento deve ser dispensado de forma a preservar compostos de interesse funcional e de qualidade em uvas passa.

De acordo com a análise multivariada, o tempo de armazenamento foi o principal fator influenciador das variáveis estudadas, independente da utilização de pré-tratamento químico.

\section{REFERÊNCIAS}

ACHKAR, M. T.; NOVAES, G. M.; SILVA, M. J. D.; VILEGAS, W. Propriedade antioxidante de compostos fenólicos: importância na dieta e na conservação de alimentos. Revista da Universidade Vale do Rio Verde, v. 11, n. 2, p. 398-406, 2013.Disponível em: http://periodicos.unincor.br/index.php/revistaunincor/article/view/1151>. http://dx.doi.org/10.5892/ruvrd.v11i2.398406.

AMARAL, G. V.; CONCEIÇÃO, R. L. C.; MACEDO, R. D.; PIRES, M.M. O Desempenho das Exportações Brasileiras de Uva: Uma Análise da Competitividade da Região do Vale do São Francisco no Período de 2005 a 2014. Revista Cadernos de Aulas do LEA, n. 5, p. 1-17, 2016. Disponível em: <http://periodicos.uesc.br/index.php/calea/article/view/1249/1047>.

AOAC - Association of Analytical Chemists. Official methods of the Association of the Agricultural Chemists. 19th edition. Whashington, 2012.

BINGOL, G.; ROBERTS, J. S.; BALABAN, M. O.; DEVRES, Y. O. Effect of Dipping Temperature and Dipping Time on Drying Rate and Color Change of Grapes. Drying Technology: An International Journal, v. 30, n.6, p. 597-606, 2012. Disponível em: <https://www.tandfonline.com/doi/abs/10.1080/07373937.2011.654020.> doi: 10.1080/07373937.2011.654020.

BONFIETTI, N. F. Desenvolvimento e análise sensorial de cookies de quinoa enriquecidos com pigmentos naturais. Revista Saúde UniToledo, Araçatuba, SP, v. 01, n. $01, \quad$ p. 31-46, 2017. Disponível em: <http://www.ojs.toledo.br/index.php/saude/article/view/87>.

BRASIL. Leis, Decretos, etc. Instrução normativa N¹ de 7 de janeiro de 2000. Regulamento técnico geral para a fixação dos padrões de identidade e qualidade para polpa de fruta. 2000. Disponível em: http://extranet.agricultura.gov.br/sislegisconsulta/consultarLegislacao.do?operacao=visualizar\&id=7777. 
BUCKOW, R.; KASTELL, A.; TEREFE, N. S.; VERSTEEG, C. Pressure and Temperature Effects on Degradation Kinetics and Storage Stability of Total Anthocyanins in Blueberry Juice. Journal of Agricultural and Food Chemistry, v. 58, n. 18, p. 10076-10084, 2010. Disponível em: <https://pubs.acs.org/doi/abs/10.1021/jf1015347>. doi: 10.1021/jf1015347.

CARNEIRO, L. M.; PIRES, C. R. F.; LIMA, J. P.; PEREIRA, P. A. P.; LIMA, L. C. O. Avaliação da estabilidade de geleias de amora-preta acondicionadas em diferentes embalagens. Journal of Bioenergy and Food Science, v.3, n.2, p. 89-102, 2016. Disponível em: <http://periodicos.ifap.edu.br/index.php/JBFS/article/view/99>. doi: 10.18067/jbfs.v3i2.99.

CARRANZA-CONCHA, J.; BENLLOCH, M.; CAMACHO, M.M.; MARTÍNEZNAVARRETE, N. Effects of Drying and Pretreatment on the Nutritional and Functional Quality of Raisins. Food and Bioproducts Processing, v. 90, n. 2, p. 243-248, $2012 . \quad$ Disponível em: <https://www.sciencedirect.com/science/article/pii/S0960308511000344>. doi: 10.1016/j.fbp.2011.04.002.

COSTA, J. D. S.; NETO, A. F.; NUNES, S. M.; RYBKA, A. C. P.; BIASOTO, A. C. T.; FREITAS, S. T. Caracterização Física e Fisico-química de Uva Itália Desidratada. Revista Iberoamericana de Tecnología Postcosecha, v. 16, n. 2, p. 273-280, 2015. Disponivel em: <http://www.redalyc.org/articulo.oa?id=81343176018>.

DEBASTIANI, G.; LEITE, A. C.; WEIBER JUNIOR, C. A.; BOELHOUWER, D. I. Cultura da Uva, Produção e Comercialização de Vinhos no Brasil: Origem, Realidades e Desafios. Revista Cesumar Ciências Humanas e Sociais Aplicadas, v.20, n.2, p. 471-485, $2015 . \quad$ Disponível em: <http://periodicos.unicesumar.edu.br/index.php/revcesumar/article/view/4395/2718>.

FREITAS, D. G. C.; MACHADO, J. A.; MATTOS, C. T. G. B.; NOGUEIRA, R. I.; CORNEJO, F. E. P.; SOUZA, R. T.; MAIA, J. D. G.; RITSCHEL, O. S. Aceitação de Uvas Passas Brasileiras e suas Características Sensoriais Segundo a Percepção do Consumidor. Circular Técnica 192. Embrapa, ISSN 0103 5231, Rio de Janeiro, RJ, 2013. Disponível em: <https://www.embrapa.br/busca-depublicacoes/-/publicacao/973261/aceitacao-de-uvas-passas-brasileiras-e-suascaracteristicas-sensoriais-segundo-a-percepcao-do-consumidor>.

GABAS, A. L.; MENEGALLI, F. C.; TELIS-ROMERO, J. Effect of Chemical Pretreatment on the Physical Properties of Dehydrated Grapes. Drying Technology: ENCICLOPÉDIA BIOSFERA, Centro Científico Conhecer - Goiânia, v.15 n.27; p.146 2018 
An International Journal, v. 17, n. 6, p. 1215-1226, 1999. Disponível em: <https://www.tandfonline.com/doi/abs/10.1080/07373939908917606>. . doi: 10.1080/07373939908917606.

GABAS, A. L.; TELIS-ROMERO, J.; MENEGALLI, F. C. Permeabilidade da Casca de Uva Itália. Brazilian Journal of Food Technology, v. 1, n. 1-2, p. 90-96, 1998. Disponível em: <http://bjft.ital.sp.gov.br/artigos/html/busca/PDF/v01nu10a.pdf>.

GIUSTI, M. M.; WROLSTAD, R. E. Characterization and Measurement with UVVisible Spectroscopy. In: Wrolstad, R. E. (Ed.). Current Protocols in Food Analytical Chemistry. New York: John Wiley \& Sons. Unit. F1.2.1-13, 2001. Disponível em: <http://www.academia.edu/6873755/Characterization_and_Measurement_of_Anthoc yanins_by_UV-Visible_Spectroscopy>.

GOUVEIA, A. M. S.; CORREIA, C. V.; TAVARES, A. E.; EVANGELISTA, R. M.; CARDOSO, A. I. I. Qualidade de Raízes de Batata-Doce em Função da Adubação Nitrogenada e Conservação. Revista Raízes e Amidos Tropicais, v. 10, n. 1, p. 5764, $2014 . \quad$ Disponível em: <http://energia.fca.unesp.br/index.php/rat/article/view/1790>. doi: http://dx.doi.org/10.17766/1808-981X.2014v10n1p57-64.

GUINÉ, R. P.; PALIYATH, G.; PESSOA, F. L.; LE QUÉRÉ, J. L.; SIDHU, J. S.; SINHA, N. Handbook of fruit and vegetable flavors. Y. H. Hui, F. Chen, \& L. M. Nollet (Eds.). John Wiley and Sons. 1118 p., 2010. Disponível em: <https://onlinelibrary.wiley.com/doi/book/10.1002/9780470622834>. doi: 10.1002/9780470622834.

JAVED, H. U.; WANG, D; SHI, Y.; WU, G. F.; XIE, H et al. Changes of free-form volatile compounds in pre-treated raisins with different packaging materials during storage. Food Research International, v. 107, p. 649-659, 2018. Disponível em: <https://www.sciencedirect.com/science/article/pii/S0963996918301868>. doi:10.1016/j.foodres.2018.03.019

JESZKA-SKOWRON, M.; ZGOLA-GRZEŚKOWIAK, A.; STANISZ, E.; WAŚKIEWICZ, A. Potential health benefits and quality of dried fruits: goji fruits, cranberries and raisins. Food Chemistry, v. 221, p. 228-236, 2017. Disponível em: <https://www.sciencedirect.com/science/article/pii/S0308814616316661>. doi: http://dx.doi.org/10.1016/j.foodchem.2016.10.049. 
JUNIOR, E. N. M.; SOARES, S. S.; GOMES, P. W. P.; RIBEIRO, C. F. A.; SILVA, R. M. V. Estudo do armazenamento da polpa do fruto ginja Eugênia uniflora L. e sua influencia nos teores de ácido ascórbico e antocianinas. Scientia Plena, v. 12, n. 06 , 2016. Disponível em: <https://www.scientiaplena.org.br/sp/article/view/3111>. doi: 10.14808/sci.plena.2016.069932.

LINS, A. R.; SARTONI, G. V. Qualidade Fenólica e Atividade Antioxidante de Vinhos Tintos Produzidos no Estado do Paraná. Revista Brasileira de Produtos Agroindustriais, v.16, n.1, p.69-76, 2014. Disponível em: <http://www.deag.ufcg.edu.br/rbpa/rev161/Art1619.pdf>.

MACHADO, A. V.; SOUZA, J. A.; NOVAES, R. S. Estudo cinético da secagem da uva Isabel para produção de uva passa. Revista Verde. Pombal - PB, Brasil, v. 10, n.1, $\quad$ p. $\quad 47 \quad-\quad 2015 . \quad 51, \quad$ Disponível em: <http://www.gvaa.com.br/revista/index.php/RVADS/article/view/3324>. doi: http://dx.doi.org/10.18378/rvads.v10i1.3324.

MASCARENHAS, R. J.; SILVA, S. M.; LOPES, J. D.; LIMA, M. A. C. Avaliação Sensorial de Uvas de Mesa Produzidas no Vale do São Francisco e Comercializadas em João Pessoa - Pb. Revista Brasileira de Fruticultura, v. 32, n. 4, p. 993-1000, 2010. Disponível em: <http://www.scielo.br/pdf/rbf/v32n4/aop15310.pdf>.

NICOLOSI, E.; FERLITO, F.; AMENTA, M.; RUSSO, T.; RAPISARDA, P. Changes in the quality and antioxidant components of minimally processed table grapes during storage. Scientia Horticulturae, v. 232, p. 175-183, 2018. Disponível em: <https://www.sciencedirect.com/science/article/pii/S0304423817307732>. doi: https://doi.org/10.1016/j.scienta.2017.12.050.

RIBEIRO, L. M. P.; DAMASCENO, K. A.; GONÇALVES, R. M. S.; GONÇALVES, C. A. A.; ALVES, A. N.; CUNHA, M. F. Acidez, sua relação com pH e qualidade de geleias e doces em barra. Boletim Técnico IFTM, ano 2, n.2, p.14-19, 2016. Disponível em: <http://editora.iftm.edu.br/index.php/boletimiftm/article/view/167/68>.

RIBEIRO, T. P.; LIMA, M. A. C.; ALVES, R. E. Maturação e Qualidade de Uvas para Suco em Condições Tropicais, nos Primeiros Ciclos de Produção. Pesquisa Agropecuária Brasileira, v.47, n.8, p.1057-1065, 2012. Disponível em: <http://www.scielo.br/pdf/pab/v47n8/47n08a05.pdf>.

ROCHA, D. S; REED, E. Pigmentos Naturais em Alimentos e sua Importância para a Saúde. Estudos Vida e Saúde, v. 41, n. 1, p. 76-85, 2014. Disponível em: 
<http://seer.pucgoias.edu.br/index.php/estudos/article/view/3366>.

doi:

http://dx.doi.org/10.18224/est.v41i1.3366.

ROTILI, M. C. C.; COUTRO, S.; CELANT, V. M.; VORPAGEL, J. A.; BARP, F. K.; SALIBE, A. B.; BRAGA, G. C. Composição, atividade antioxidante e qualidade do maracujá amarelo durante armazenamento. Semina: Ciências Agrárias, Londrina, v. $34, \quad$ n. $1, \quad$ p. 227-240, 2013. Disponível em: <http://www.uel.br/revistas/uel/index.php/semagrarias/article/view/12542>. doi: 10.5433/1679-0359.2013v34n1p227.

RUFINO, M. S. M.; ALVES, R. E.; BRITO, E. S.; MORAIS, S. M.; SAMPAIO, C. G.; JIMENEZ, J. P.; CALIXTO, F. D. S. Determinação da atividade antioxidante total em frutas pela captura do radical livre DPPH. Comunicado Técnico Embrapa, 127: 1-4, 2007. Disponível em: https://www.embrapa.br/agroindustria-tropical/busca-depublicacoes/-/publicacao/426953/metodologia-cientifica-determinacao-da-atividadeantioxidante-total-em-frutas-pela-captura-do-radical-livre-dpph.

RYBKA, A. C. P.; FREITAS, S. T.; NETTO, A. F.; BIASOTO, A. C. T. Central composite rotatable design approach to optimize 'Italia' raisin drying conditions. Comunicata Scientiae, v. 6, n. 4, p. 454-462, 2015. Disponível em: https://comunicatascientiae.com.br/comunicata/article/view/993. 10.14295/CS.v6i4.993.

SANTOS, A. C. A.; MARQUES, M. M. P.; SOARES, A. K. O.; FARIAS, L. M.; FERREIRA, A. K. A.; CARVALHO, M. L. Potencial antioxidante de antocianinas em fontes alimentares: revisão sistemática. Revista Interdisciplinar, v. 7, n. 3, p. 149156, 2014.2 Disponível em: <http://revistainterdisciplinar.uninovafapi.edu.br/index.php/revinter/article/view/467>.

SANTOS, E. H. B.; AZEVÊDO, L. C.; BATISTA, F. P. R.; MATOS, L. P.; LIMA, M. S. Caracterização química e sensorial de uvas desidratadas, produzidas no Vale do São Francisco para infusão. Revista Semiárido De Visu, v.1, n.2, p.134-147, 2011. Disponível em: $<$ https://periodicos.ifsertaope.edu.br/ojs2/index.php/revista/article/view/67>.

SCHIOZER, A. L.; BARATA, L. E. S. Estabilidade de Corantes e Pigmentos de Origem Vegetal. Revista Fitos, v. 3, n. 02, p.6-24, 2007. Disponível em: http://www.revistafitos.far.fiocruz.br/index.php/revista-fitos/article/view/71. 
SÉRIO, S.; RIVERO-PÉREZ, M. D.; CORREIA, A. C.; JORDÃO, A. M.; JOSÉ, M. L. G. Analysis of Commercial Grape Raisins: Phenolic Content, Antioxidant Capacity and Radical Scavenger Activity. Ciência e Técnica Vitivinícola, v. 29, n. 1, p. 1-8, $2014 . \quad$ Disponível em: <https://www.ctv-jvejournal.org/articles/ctv/abs/2014/01/ctv20142901p1/ctv20142901p1.html>. . doi: https://doi.org/10.1051/ctv/20142901001.

SILVA, A. C. G. C.; FONTES, C. H. O.; BARBOSA, A. S. Multicriteria evaluation model for organizational performance management applied to the Polo Fruit Exporter of the São Francisco Valley. Computers and Electronics in Agriculture, v. 117, p. 168-176, $2015 . \quad$ Disponível em: <https://www.sciencedirect.com/science/article/pii/S016816991500229X>. doi: http://dx.doi.org/10.1016/j.compag.2015.08.003.

SINGLETON, V. L.; ORTHOFER, R.; LAMUELA-RAVENTOS, R. M. Analysis of total phenols and other oxidation substrates and antioxidants by means of Folin-Ciocalteu reagent. Method Enzymol, 299, 152-178, 1999. Disponível em: <https://www.sciencedirect.com/science/article/pii/S0076687999990171>. doi: https://doi.org/10.1016/S0076-6879(99)99017-1.

SOUZA, R. T.; CORNEJO, F. E. P.; NOGUEIRA, R. I.; FREITAS, D. G. C.; PROTAS, J. F. S.; MAIA, J. D. G.; MATTOS, C. T. G. B.; LEAL JUNIOR, W. F.; RITSCHEL, P. S. Uvas-passas Brasileiras: Matéria-prima e Processamento. Circular Técnica 115. Embrapa, ISSN 1516-5914, Bento Gonçalves, RS, 2015. Disponível em: $<$ https://www.embrapa.br/uva-e-vinho/busca-de-

publicacoes/-/publicacao/1014820/uvas-passas-brasileiras-materia-prima-eprocessamento>.

TARHAN, S. Selection of chemical and thermal pretreatment combination for plum drying at low and moderate drying air temperatures. Journal of Food Engineering, v. $79, \quad$ n. $1, \quad$ p. 255-260, 2007. Disponível em: <https://www.sciencedirect.com/science/article/pii/S0260877406001361>. doi:10.1016/j.jfoodeng.2006.01.052.

UDOMKUN, P.; NAGLE, M.; ARGYROPOULOS, D.; MAHAYOTHEE, B.; LATIF, S.; MULLER, J. Compositional and functional dynamics of dried papaya as affected by storage time and packaging material. Food Chemistry, v. 196, p. 712-719, 2016. Disponível em: <https://www.sciencedirect.com/science/article/pii/S0308814615014557>. http://doi.org/10.1016/j.foodchem.2015.09.103. 
VILAS BOAS, A. C.; HENRIQUE, P. C.; LIMA, L. C. O.; NETO, A. C.; NASSUR, R. C. M. R.; LIMA, R. A. Z. Atividade antioxidante e fenólicos totais em blends de sucos de uvas americanas 588 produzidas no sudoeste de Minas Gerais. Centro de Pesquisa e Processamento de Alimentos. v. 34, n. 1, p. 15-26, 2016. Disponível em: <http://revistas.ufpr.br/alimentos/article/view/48969>. doi: http://dx.doi.org/10.5380/cep.v34i1.48969.

ZHANG, H.; TSAO, R. Dietary polyphenols, oxidative stress and antioxidant and antiinflammatory effects. Current Opinion in Food Science, v. 8, p. 33-42, 2016. Disponível em: <https://www.sciencedirect.com/science/article/pii/S2214799316300133>. doi: http://dx.doi.org/10.1016/j.cofs.2016.02.002. 